\title{
Alternativa de biorremediación a partir de residuos de cacao en la obtención de hongos Pleurotus ostreatus con la implementación de un análisis multicriterio
}

\author{
Juan E. Ortiz; Yisel Mejía; David E. González; Luz S. García-Alzate; Ximena Cifuentes-Wchima \\ Universidad La Gran Colombia, Facultad de Ingenierías, Grupo de Investigación Gerencia de la Tierra, Campus \\ Ciudadela del Saber La Santa María, km 7 vía \\ Armenia, La Tebaida. \\ *garciaalzluz@miugca.edu.co
}

Fecha recepción: Octubre 15 de 2019 Fecha aceptación: enero 17 de 2020

\begin{abstract}
Resumen
La producción de cacao Theobroma cacao L. en Colombia evidencia un crecimiento promedio de 6,48 \% en los últimos 10 años, lo cual ha desencadenado problemáticas ambientales, debido a que solo el $10 \%$ del fruto es utilizado para la industria agronómica. El objetivo de esta investigación fue aprovechar el residuo derivado de la producción de cacao en la obtención de hongos Pleurotus ostreatus como técnica de biorremediación con la implementación de un análisis multicriterio del cultivo. La cáscara se caracterizó fisicoquímicamente con los parámetros de $\mathrm{pH}$, lignina y celulosa, entre otros. Posteriormente, se empleó como sustrato para el cultivo de hongos y se realizó la evaluación del flujo de lixiviados del cultivo a través del análisis multicriterio con ponderaciones de variables físico-ambientales. La caracterización fisicoquímica de la cáscara reportó un $\mathrm{pH}$ de $5,47 \pm 0,004$; un porcentaje de humedad del $84,345 \pm 0,002$

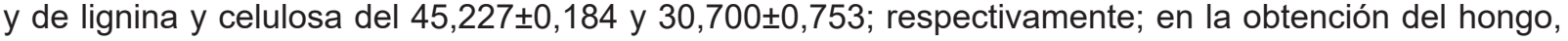
se observó la aparición de los primordios a los 16 días y del hongo para cosechar a los 21 días, lo cual redujo los residuos en un $60 \%$. En el análisis multicriterio, las ponderaciones arrojaron un flujo medio de lixiviados en el tercer trimestre del año 2018, lo que indica que los lixiviados fluyen por medio del drenaje hidrológico. Para concluir, la caracterización y el crecimiento del hongo determinan que la cáscara funciona como sustrato para la obtención de hongos Pleurotus ostreatus, y es una alternativa de biorremediación de uso agronómico, donde la información del análisis multicriterio permite decisiones oportunas sobre el cultivo.
\end{abstract}

Palabras clave: Biorremediación; Producción; Lixiviados; Multicriterio; Residuo. 


\title{
Alternative bioremediation from cocoa residues in obtaining Pleurotus ostreatus fungi with the implementation of a multicriteria analysis
}

\begin{abstract}
Theobroma cacao L. cocoa production in Colombia shows an average growth of $6.48 \%$ in the last 10 years; triggering environmental problems, because only $10 \%$ of the fruit is used for the agronomic industry. The objective of this research was to take advantage of the residue derived from cocoa production in obtaining Pleurotus ostreatus fungi as a bioremediation technique with the implementation of a multicriteria crop analysis. The shell was characterized physicochemically with the parameters of $\mathrm{pH}$, lignin and cellulose, among others. Subsequently, it was used as a substrate for the cultivation of fungi and the evaluation of the leachate flow of the culture was carried out through multicriteria analysis with weights of physical-environmental variables. The physicochemical characterization of the shell reported a pH of $5.47 \pm 0.004$, a moisture percentage of 84.345 \pm 0.002 and of lignin and cellulose of $45.227 \pm 0.184$ and $30.700 \pm 0.753$, respectively; in obtaining the fungus, the appearance of the primordia was observed at 16 days and the fungus to harvest at 21 days, reducing waste by $60 \%$. In the multicriteria analysis, the weights showed an average flow of leachate in the third quarter of 2018, indicating that leachate flows through hydrological drainage. To conclude, the characterization and growth of the fungus determine that the shell works as a substrate for obtaining Pleurotus ostreatus fungi, being an alternative of bioremediation for agronomic use, where the information from the multicriteria analysis allows timely decisions on the crop.
\end{abstract}

Keywords: Bioremediation; Production; Leachate; Multicriteria; Residue.

\section{Biorremediação alternativa a partir de resíduos cacaueiros na obtenção de fungos Pleurotus ostreatus com a implementação de uma análise multicritério}

\begin{abstract}
Resumo
Produção de cacau Theobroma cacao L. na Colômbia mostra um crescimento médio de 6,48 \% nos ultimos 10 anos; desencadeando problemas ambientais, porque apenas $10 \%$ da fruta é utilizada na indústria agronômica. O objetivo desta pesquisa foi aproveitar o resíduo derivado da produção de cacau na obtenção de fungos Pleurotus ostreatus como técnica de biorremediação com a implementação de uma análise multicritério de colheita. Aconcha foi caracterizada físico-quimicamente com os parâmetros de $\mathrm{pH}$, lignina e celulose, entre outros. Posteriormente, foi utilizado como substrato para o cultivo de fungos e a avaliação do fluxo de lixiviados da cultura foi realizada por meio de análise multicritério com pesos das variáveis físico-ambientais. A caracterização físico-química da concha relatou um $\mathrm{pH}$ de 5,47 $\pm 0,004$, porcentagem de umidade de 84,345 $\pm 0,002$ e lignina e celulose da 45,227 \pm 0,184 e 30,700 \pm 0,753; respectivamente; na obtenção do fungo, observou-se o aparecimento dos primórdios aos 16 dias e o fungo a colher aos 21 dias, reduzindo o desperdício em $60 \%$. Na análise multicritério, os pesos mostraram um fluxo médio de lixiviado no terceiro trimestre de 2018, indicando que o lixiviado flui através da drenagem hidrológica. Concluindo, a caracterização e crescimento do fungo determinam que a concha funcione como substrato para a obtenção de fungos Pleurotus ostreatus, sendo uma alternativa de biorremediação para uso agronômico, onde as informações da análise multicritério permitem decisões oportunas sobre a colheita.
\end{abstract}

Palavras-chave: Biorremediação; Rodução; Lixiviado; Multicritério; Resíduo. 


\section{Introducción}

El cacao Theobroma cacao L. en Colombia se ha venido desarrollando notablemente. Esto se ve reflejado en los datos de producción del 2018, año en que se generaron 56.000 toneladas métricas, lo cual evidencia un crecimiento promedio de 6,48 \% en los últimos 10 años [1]. Por otro lado, desde un punto de vista local, el Quindío produjo 156 toneladas métricas en el 2018, según lo reportado por la Federación Nacional de Cacaoteros en sus informes nacionales; a raíz de estas prelaciones, el Gobierno nacional ha considerado el cacao como un producto de exportaciones internas y externas, debido a que este fruto se encuentra dentro los productos que conforman el $21 \%$ de las exportaciones netas del país, provenientes del sector agrícola, donde se encuentran otros cultivos importantes, como lo son los cultivos de bananos, plátanos, arroz, algodón, tabaco y caña de azúcar [2]. Ahora bien, todos estos cultivos generan impactos ambientales que pueden llegar a ocasionar aumento de gases efecto invernadero, erosión de suelos o generación de residuos sólidos de origen vegetal. Esto genera una contaminación de la atmósfera y de los cuerpos de agua cercanos, de los cuales se benefician las poblaciones cercanas a estos cultivos [3]. En el caso del cacao, los reportes de producción antes mencionados representan solo el $10 \%$ de lo que se deriva del cultivo de este fruto, y generan el $90 \%$ en residuos como lo son la cáscara y el mucílago. A este último, comúnmente, no se le encuentra una utilidad eficiente, más que para ser utilizado como abono, lo cual no resulta viable debido a la lenta degradación del sustrato que, acumulado en grandes cantidades, afecta directamente la calidad del fruto y los resultados de la producción del cultivo. Debido a enfermedades como $P$. nicotianae var. parasitica, Moniliophthora perniciosa, la moniliasis (Moniliophthora roreri), las cuales pueden llegar a generar daños en el cultivo del 40 al $100 \%$ [4]. Esto significa como mínimo la pérdida de 2 a 4 años de labor, ya que este es el tiempo que demora en producirse la primera cosecha de cacao, según el Centro Agronómico Tropical de Investigación y Enseñanza (CATIE) [5]. Lo anterior desencadena deficiencias económicas para el agricultor. Es por ello que la descomposición descontrolada de estos residuos puede ocasionar propagación de microorganismos patógenos, roedores o insectos al cultivo y enfermedades para la población, lo cual genera costos innecesarios para los productores al intentar mitigar estos impactos de manera tradicional.

Paralelamente, en el proceso de degradación del sustrato se derivan los lixiviados, los cuales son sustancia líquidas, que presentan una composición química similar a la del sustrato. Al respecto, autores como Najera [6] reportan que los residuos en descomposición y el vertimiento de los lixiviados impactan directamente sobre la condición del suelo, pues este es el soporte y fuente de los nutrientes necesarios para el adecuado desarrollo de las plantas. Al mismo tiempo, afectan el hábitat de una gran variedad de micro y macroorganismos, que permiten regular la retención de carbono, la emisión de gases efecto invernadero, las dinámicas de la materia orgánica y los regímenes de agua [7]. Es por ello que la saturación del suelo con las sustancias derivadas del cultivo amenaza la conservación de estos organismos necesarios para un adecuada calidad del suelo. Además de esto, los lixiviados no son un fenómeno estático, debido a factores externos, como los geomorfológicos (relieve) y climáticos (precipitaciones), lo cual genera que los lixiviados sean arrastrados por medio del drenaje hidrológico hacia los cuerpos de agua cercanos y a otros cultivos adyacentes al sector [6]. Según estos autores, en la actualidad, se desconocen las utilidades del posproceso de la producción del cacao y al mismo tiempo la generación de lixiviados debido a su descomposición. Por tanto, es relevante la realización de la caracterización de la cáscara de cacao, ya que permite definir cuáles son las opciones más viables para aprovecharla.

Es por todo lo anterior que es una alternativa viable utilizar la especie Pleurotus ostreatus como agente biorremediador, teniendo en cuenta su capacidad para alimentarse y descomponer biopolímeros, como la lignina y la celulosa, los cuales se encuentran presentes en la cáscara de cacao que resulta de la posproducción. Mediante el aprovechamiento de este residuo se evita su descomposición descontrolada, al tiempo que se evita la generación de lixiviados que puedan producir enfermedades tanto en el cultivo de cacao como en otros cultivos cercanos a este, o en las comunidades establecidas en sus alrededores [8]. De igual forma, el consumo de hongos del género Pleurotus trae consigo diferentes ventajas para la salud humana. Sobre este tema, Centro Nacional de Investigaciones de Café - Cenicafe ha declarado que el compuesto Pleurotin (policíclico) permite que el hongo tenga propiedades antibióticas. Así mismo, estos hongos presentan un gran potencial 
como agente controlador biológico de nematodos (organismos pluricelulares con un tamaño inferior a $2 \mathrm{~mm}$ de largo) [9], al igual que de bacterias del género Agrobacterium y Pseudomonas [10].

Por otro lado, el uso del Sistema de Información Geográfico (SIG), un instrumento integrado de hardware, software y datos, permite visualizar información geográfica a través de sistemas organizados, debido a que procesa, de manera interdisciplinar, diferentes datos alfanuméricos y espaciales, deforma precisa y eficiente, considerando aspectos como el relieve, los polígonos de estudio, la precipitación, el drenaje, entre otros, para lograr una adecuada toma de decisiones. Por lo tanto, la capacidad de análisis que facilita una herramienta como SIG, denota de manera precisa las dinámicas espaciales complejas relacionadas con el flujo de lixiviados, en el cual su capacidad de propagación y dirección es influenciada por factores meteorológicos y topográficos, respectivos a la ubicación del cultivo; en consecuencia, esta información presta una ayuda para la toma de decisiones acertadas sobre la actividad realizada, teniendo en cuenta lo establecido por Prieto y Martínez [11].

Por tanto, además de emplear el residuo de cacao en la producción de hongos Pleurotus ostreatus como agente biorremediador; se implementó la herramienta SIG con el fin de analizar e interactuar con la información relacionada con el flujo de lixiviados de un cultivo de Theobroma cacao L., a través de la manipulación de las debidas capas, la asignación de atributos y la ponderación de las variables, lo cual proporciona una modelación y consulta de los datos obtenidos en el mapa, con la intención de divulgar esta información a las entidades públicas correspondientes $\mathrm{o}$ a los productores que estén interesados en mitigar los impactos que pueden estar generando estos residuos sobre el ambiente y la calidad del cultivo [11].

El objetivo de esta investigación fue aprovechar el residuo derivado de la producción de cacao en la obtención de hongos Pleurotus ostreatus como técnica de biorremediación con la implementación de un análisis multicriterio del cultivo.

\section{Materiales y métodos}

\section{Obtención del residuo vegetal (cáscara de cacao)}

Los subproductos del cacao se obtuvieron de productores del Quindío, específicamente de la finca Paraná, en el municipio de la Tebaida; con coordenadas $75^{\circ} 47^{\prime} 41,047^{\prime \prime}$ W y $4^{\circ} 24^{\prime} 47,238^{\prime \prime} \mathrm{N}$.
Las determinaciones en esta investigación se llevaron a cabo en los laboratorios de la Facultad de Ingenierías Ciudadela del Saber, campus La Santa María, Universidad La Gran Colombia.

\section{Preparación de la muestra}

Las cáscaras de cacao fueron cortadas en láminas, y después se les realizó un troceado con una picadora marca Penagos TP-8R, para así tener un tamaño de muestra más pequeña y lograr establecer las variables fisicoquímicas de la caracterización de manera más eficiente, además de obtener una mejor invasión por parte del micelio en la producción de hongos.

\section{Caracterización fisicoquímica del residuo vegetal (cáscara de cacao)}

Se realizó el análisis proximal del sustrato donde se determinaron los parámetros de $\mathrm{pH}$, humedad, cenizas, grasa, fibra, lignina y celulosa, teniendo en cuenta las siguientes normas y técnicas:

Para establecer el pH se utilizó un potenciómetro según la norma NMX-AA-25 [12]. El porcentaje de humedad se midió empleando una estufa de convección forzada ESCO de acuerdo a la norma AOAC 20,013 [13]. Para el parámetro de cenizas se empleó una mufla Terrígeno para calcinación de la muestra utilizando la metodología establecida en la norma TAPPI 211 om-02 [14]. La determinación del porcentaje de fibra se realizó por medio de extracción Soxhlet de acuerdo a la norma AOAC 962,09 [15]. Igualmente, la variable de porcentaje de grasa se obtuvo mediante la extracción Soxhlet reportada en la norma NTC 1142 [16], el porcentaje de lignina, según lo establecido por la norma TAPPI 222 om88 [17], y la variable de porcentaje de celulosa se realizó empleando la norma NTC 697 [18].

\section{Producción del hongo Pleurotus ostreatus}

Para la siembra del cuerpo fructífero, se empleó el sustrato de cáscara de cacao para la obtención de hongos, con una siembra en capas de sustratosemilla a una concentración de semilla al 4,0\%, basado en lo establecido por Toledo [19] y García et al. [20]. Posteriormente, se realizó la cosecha del hongo de forma manual, extrayendo el cuerpo fructífero desde su base.

El sustrato de cáscara de cacao se desinfectó a temperatura ambiente por medio de una inmersión de solución al $0,6 \%$ de carbonato de calcio durante 13 días, teniendo en cuenta lo propuesto por Rodríguez y Jaramillo [10]. Después de esto, se acondicionó la humedad relativa al 70-90 \%, pH 
de 5,0-7,0, para procurar el óptimo desarrollo del hongo. Para la inoculación, se realizó la mezcla del micelio con cada uno de los sustratos en condiciones óptimas de asepsia y se empacaron en 1000 gramos de sustrato por bolsa con un $4,0 \%$ de semilla. Posterior a esto, se realizó la incubación a un rango de temperatura de 24$28{ }^{\circ} \mathrm{C}$ y un rango de humedad relativa de $70-80$ $\%$, para garantizar las condiciones ambientales óptimas de oscuridad y baja ventilación para el crecimiento del hongo, con monitoreo de estas variables. Terminada la etapa de incubación, las bolsas se expusieron a la luz natural, para inducir la formación del hongo, monitoreando la cantidad de luz, la humedad relativa y la temperatura. La recolección del hongo se realizó desprendiendo los cuerpos fructíferos de la bolsa cuando la parte superior del hongo (píleo) estaba casi plano, de acuerdo a lo indicado por García et al. [20].

\section{Implementación de análisis multicriterio para la evaluación de flujo de lixiviados}

Para la evaluación del flujo de lixiviados, se realizó un modelado del drenaje sencillo al utilizar un modelo digital de elevación (MDE), por medio de las curvas de nivel de la cartografía base del Quindío, con una precisión de $10 \mathrm{~m}$, definiendo el drenaje hidrológico del sector. Después de esto, se procedió a la ponderación de la variables, al establecer las dinámicas geográficas y los datos alfanuméricos entre volumen de desechos en el cultivo y la precipitación del sector. Se logró visualizar las influencias hidrológicas (precipitación) y geomorfológicas (relieve) que recaen sobre el transporte de los lixiviados, a través del uso del software ArcGis 10,5 de la compañía ESRI, teniendo en cuenta la metodología propuesta por Bolaños y Rojas [21][21], y a partir de los rangos de precipitación mostrados en la tabla 1, la cual representa la relación de ponderación espacial que existe entre la variable de precipitación (valores alfanuméricos - cuantitativos), con base en una producción media del cultivo por trimestre.

Tabla 1. Rangos de precipitación en el Quindío.

\begin{tabular}{cc}
\hline Precipitación $(\mathbf{m m})$ & Rango \\
\hline$<80$ & Bajo \\
$80-169$ & Medio \\
$169-305$ & Alto \\
\hline
\end{tabular}

Seguido de esto se empleó la información depositada en la tabla 2, la cual corresponde a la ponderación completa al combinar los rangos mostrados de la tabla 1 y la producción media del cultivo de cada uno de los trimestres. Finalmente, se generó el resultado de flujo de lixiviados presentes en el cultivo de cacao, con base en la tasa de precipitación y, al mismo tiempo, considerando la tasa de producción dada por los agricultores.

Tabla 2. Ponderación de las variables.

\begin{tabular}{ccc} 
Precipitación & $\begin{array}{c}\text { Producción } \\
\text { por trimestre }\end{array}$ & Flujo de lixiviado \\
\hline Baja & Baja & Flujo muy bajo \\
Baja & Media & Flujo bajo \\
Media & Media & Flujo medio \\
Media & Alta & Flujo alto \\
Alta & Alta & Flujo muy alto \\
\hline
\end{tabular}

\section{Análisis estadístico}

Se realizó un estudio de la significancia de los diferentes efectos en la cáscara de cacao. Las variables medidas fueron $\mathrm{pH}$, humedad, cenizas, grasa, fibra, lignina y celulosa. Se realizó un análisis de la varianza (ANOVA), los resultados con un nivel de significancia $(\alpha)$ de 0,05 , con el paquete estadístico Statgraphics Centurión XV (Versión 15.2.05).

\section{Resultados y discusión}

\section{Caracterización fisicoquímica del residuo vegetal (cáscara de cacao)}

Las variables determinadas en la caracterización fisicoquímica del residuo vegetal (cáscara de cacao Theobroma cacao L.) se pueden observar en la tabla 3.

Tabla 3. Caracterización fisicoquímica del residuo vegetal Theobroma cacao L.

\begin{tabular}{cc}
\hline $\begin{array}{c}\text { Variable } \\
( \pm \mathrm{D} . S)\end{array}$ & $\begin{array}{c}\text { Residuo Vegetal } \\
\text { (Cáscara de cacao) }\end{array}$ \\
\hline $\mathrm{pH}$ & $5,46 \pm 0,045$ \\
Humedad (\%) & $84,35 \pm 0,002$ \\
Cenizas (\% bs) & $8,23 \pm 0,003$ \\
Grasa (\% bs) & $0,07 \pm 0,004$ \\
Fibra (\% bs) & $6,73 \pm 0,012$ \\
Lignina (\% bs) & $45,23 \pm 0,184$ \\
Celulosa (\% bs) & $30,70 \pm 0,753$ \\
\hline
\end{tabular}

Número de muestras: 3; ( $\pm \mathrm{DS})$ : desviación estándar; bs: base seca

En la tabla 3 se pueden observar las variables determinadas para el residuo vegetal cáscara de cacao, donde el valor de $\mathrm{pH}$ correspondió a $5,46 \pm 0,045$, lo que indica que este residuo de 
cáscara de cacao estaba ligeramente ácido; por lo tanto, la degradación de los residuos fue más lenta, debido a que la descomposición resulta más fácil en un medio alcalino, como se establece en [22]. Además de esto, el porcentaje de humedad obtenido fue de $84,35 \pm 0,002$, lo cual significa que este residuo contiene una cantidad de agua más significativa; por lo tanto, permitió que el hongo Pleurotus ostreatus realizara una asimilación de los nutrientes de forma más efectiva, gracias a que dicho porcentaje alto de humedad posibilita metabolizar. Estos resultados se pueden comparar con el estudio de Torres [23], que describe una caracterización al mismo tipo de biomasa lignocelulósica (Theobroma cacao L.), y reporta una humedad de $81,17 \%$ y $82,04 \%$ para utilización de este material en la obtención de etanol por vía fermentativa. Además, esto es similar a lo indicado por García et al. [20], en donde se investigó sobre residuos orgánicos para la producción de hongos Pleurotus ostreatus, y se estableció que una humedad superior a $70 \%$ permite una mayor invasión por parte del hongo.

En cuanto al parámetro de cenizas $(8,23 \pm 0,003)$, este se encontró dentro del rango establecido por Sangronis et al. [24], quienes analizaron la cáscara de cacao como material de infusiones, y reportaron un rango de 7,0 al $8,0 \%$ de cenizas, lo cual concuerda con el valor expresado en la tabla 3. Además de esto, autores como García et al. [20] reportaron que el contenido de cenizas debe estar entre 6,45 y 9,13 , para tener un aporte de minerales para el desarrollo fructífero del cuerpo.

De igual forma, el porcentaje de grasa que presentó el residuo vegetal tratado fue de 0,07 \pm 0,004 , lo cual es similar a los resultados obtenidos en Villamizar y López [25], en donde se realizó una investigación sobre la cáscara de cacao como fuente de polifenoles y fibra. Esto, a su vez, concuerda con el porcentaje de fibra $(6,73 \pm 0,012)$ que contiene la cáscara de cacao, componente de gran importancia, debido a que le permite al hongo ser considerado como un alimento funcional, es decir, que en su contenido presenta ingredientes activos que traen beneficios para el cuerpo humano, principalmente en la salud, como lo expresado en [26].

Debido a que la cáscara de cacao se encuentra clasificada como un material maderero de carácter rígido, como se menciona en [27], es posible observar un alto porcentaje de lignina $(45,23 \pm$ 0,184 ) en este tipo de material vegetal con rigidez composicional.
Además, teniendo en cuenta lo expuesto por Song [28], quien estableció un porcentaje superior al $15 \%$ para el desarrollo del hongo en un cultivo tipo ostra. Este resultado es similar al obtenido en [23], donde se menciona un contenido de este componente entre 33 y $45 \%$, el cual es dependiente del tamaño de partícula empleado en esta determinación, con el fin de utilizar la cáscara de cacao como materia prima para la producción de etanol y lograr que la lignina, mediante una hidrólisis de polisacáridos, permitiera la producción de bioetanol. Sin embargo, si el porcentaje de lignina es muy alto, se presenta mayor dificultad para realizar la extracción de biopolímeros de tipo celulósico del residuo, el cual, según Torres [23], es el caso de la cáscara de cacao. Esto ocasiona que la descomposición sea más lenta, principalmente debido a su estructura. En [29] se describe una investigación acerca de la estructura de la lignina y sus aplicaciones en la obtención de derivados aromáticos de uso industrial, y se encontró que este compuesto refuerza las paredes celulares, pues forma biopolímeros con capacidades biofísicas, al igual que aumenta la resistencia del material para hidrólisis ácidas.

Además de esto, en [30], se caracterizó la fibra dietaria de la cáscara de semillas de cacao Theobroma cacao L. para una industria chocolatera colombiana, buscando mejoras en el producto; en esa investigación, se estableció que el aumento de la presencia de lignina en la cáscara depende del estado de maduración del fruto. Por lo tanto, si se busca aprovechar este biopolímero, como lo es en el caso de la reproducción del Pleurotus ostreatus, es relevante considerar el tiempo de madurez del cacao.

La variable lignina, uno de los componentes principales en la estructura química de la cáscara de cacao, genera mayor tiempo de degradación del sustrato. Además de su forma cónica como producto del proceso del descocote de la semilla la cáscara permite la acumulación de agua dentro de ella, lo cual aumenta la probabilidad de aparición de enfermedades y facilita el desarrollo de patógenos, como la Moniliophthora rorer, que pueden reproducirse en una lámina de agua y afectar la calidad del cultivo, como lo menciona Jaimes y Aranzazu [4]. Esto impacta en la salud de las plantas, y, en consecuencia, produce pérdidas para los productores de cacao. Por lo tanto, el valor de lignina de este residuo vegetal es uno de los resultados que puede producir mayor cantidad de problemáticas en un cultivo de cacao, 
por su ralentizada degradación descontrolada. No obstante, al mismo tiempo, es uno de los compuestos con mayor potencial de uso, como lo mencionan autores como García et al. [20] y Chávez y Domine [29]. Así, se aumentan las probabilidades de poder utilizar la cáscara de cacao como un sustrato viable para el hongo Pleurotus ostreatus, dándole un uso viable y funcional a este biopolímero, que no es considerado aún como una sustancia aprovechable.

Por otra parte, en la tabla 3 se puede observar la determinación del contenido de celulosa, la cual reportó valores de 30,70 $\pm 0,753$ en la cáscara de cacao. La celulosa es el principal compuesto de la pared celular del residuo vegetal utilizado y es uno de los componentes sumamente necesarios para el crecimiento del hongo, debido a que, en conjunto con la lignina, son fuente de carbono, nitrógeno y energía. A su vez, representa una de las sustancias químicas más complejas para degradar, acción lograda únicamente por hongos (género Pleurotus, Lentinus, Valvariel/a y Auricularia) y algunas Bacterias, gracias a que cuentan con enzimas con la capacidad de romper dichas moléculas [31]. Por lo tanto, los valores obtenidos del contenido de celulosa en la presente investigación $(30,70 \pm 0,753)$ superan el mínimo (25\%) para un adecuado desarrollo del Pleurotus ostreatus, como lo establece Benavides [32], quien realizó una investigación acerca del aprovechamiento de los residuos lignocelulósicos.
Investigaciones realizadas por autores como Benavides [23] obtuvieron valores de celulosa entre 21,20 y $27,04 \%$, los cuales son menores a los obtenidos en esta investigación $(30,70 \pm$ 0,753 ), debido posiblemente

a la degradación de azúcares y, a su vez, a la descomposición de estos para la generación de dos compuestos derivados del furano: el furfural, que se forma a partir de las pentosas (xilosa), y el 5-hidroximetilfurural, que se forma a partir de las hexosas (glucosa), como se establece en [33].

Los biopolímeros de lignina y celulosa presentes en el residuo vegetal (cáscara de cacao) se encargan de dar resistencia a los ataques físico, químico y biológico de la biomasa, los cuales pueden ser utilizados para el crecimiento de hongos Pleurotus ostreatus, debido a que este tipo de sistema biológico cuenta con enzimas capaces de degradar material lignocelulósico, como se estableció en [10], [20] y [31], en donde se incluyeron además otras variables requeridas para este fin. Así se genera la posibilidad de reducir volumen de este residuo por medio de la vinculación de alternativas de biorremediación.

\section{Producción del hongo Pleurotus ostreatus}

En la figura 1 se puede observar la producción de hongos Pleurotus ostreatus, cultivados a partir del sustrato vegetal cáscara de cacao (Theobroma cacao L.).

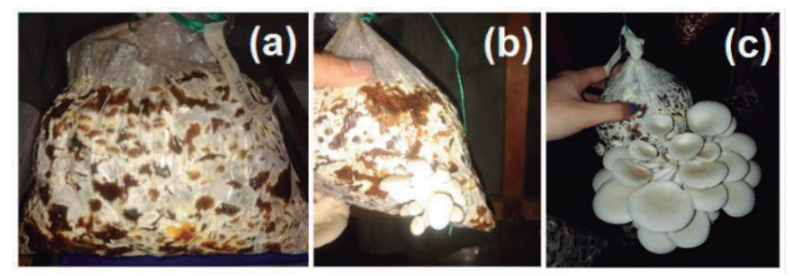

Figura 1. Producción de hongos Pleurotus ostreatus, cultivados sobre cáscara de cacao. Donde (a) corresponde a la invasión del micelio, (b) a la aparición del primordio y (c) al desarrollo del cuerpo fructífero.

De acuerdo con la figura anterior, es posible observar en (a) la primera manifestación del hongo con la invasión del micelio a través de una capa algodonosa, lo cual indica la capacidad del hongo de adaptarse en este sustrato; en segundo lugar, (b) muestra la aparición de primordios del hongo Pleurotus ostreatus, lo cual permite establecer que posiblemente se puede llegar a obtener el desarrollo final del cuerpo fructífero; en (c) se demuestra la formación de las setas producto de la asimilación de nutrientes por parte del hongo sobre este sustrato. Con lo anterior se puede manifestar que la cáscara de cacao Theobroma cacao $\mathrm{L}$. funciona como sustrato para llevar a cabo la producción de hongos ostra, como se evidencia en la figura 1.

Los resultados obtenidos en esta investigación mostraron una aparición de primordios a los 16 días. Este valor se compara a lo que obtuvieron autores como Vargas et al. [34], quienes utilizaron diferentes muestras lignocelulósicas como son bagazo de caña y hojarasca de roble, reportando una aparición de primordios a los 16 y 20 días, respectivamente; siendo similar al comparar 
con el bagazo de caña, en cuento al sustrato de hojarasca de roble, este reportó mayor tiempo de aparición de primordios (20 días), esto debido posiblemente a la cantidad de la lignina presente en la muestra, la cual genera mayor rigidez en la estructura, dificultando la colonización por parte del micelio. Finalmente, se obtuvo un desarrollo del cuerpo fructífero a los 21 días; permitiendo a su vez, generar indicios de la viabilidad de la cáscara de cacao, como sustrato para el cultivo de hongos. Por otro lado, en [35] se evaluó el crecimiento de hongos Pleurotus ostreatus en sustratos de cáscara de papa, bagazo de caña y cebada hidratada, y se logró determinar la influencia del $\mathrm{pH}$ como factor en el rendimiento de los sustratos. En esa investigación, se estableció que el pH más adecuado para un óptimo crecimiento del hongo debe ser de 5.0, resultado que se tuvo en cuenta en este trabajo por ser similar al obtenido en la cáscara de cacao (tabla 3). Este parámetro es importante debido a que el $\mathrm{pH}$ del medio de cultivo donde crece un hongo tiene una influencia directa sobre este, porque incide sobre el carácter iónico del medio e influye directamente sobre las proteínas de la membrana y sobre la actividad de las enzimas ligadas a la pared celular, es decir, que afecta su metabolismo [35].

Es por ello que, aunque el sustrato cuente con componentes lignocelulósicos presentes en su estructura, o estos sean adicionados mediante suplementos, y que durante el cultivo se realice un control de variables físicas como humedad relativa y temperatura, si el rango de $\mathrm{pH}$ del sustrato utilizado no es el adecuado, esto genera interferencias en la invasión por parte del micelio, alteraciones en el desarrollo del hongo y, finalmente, impide una adecuada producción del cultivo. Por tanto, esta es una variable que se debe considerar en el proceso. Esto es similar a lo sugerido en [36], donde se expone que el $\mathrm{pH}$ adecuado debe estar entre 4,0 y 6,5 , lo cual demuestra nuevamente que el sustrato de cáscara de cacao es viable para el desarrollo del hongo. Este hongo genera adicionalmente una adaptabilidad a medios con alto contenido de carbohidratos, cuyos rendimientos permiten obtener niveles de producción adecuados en crecimiento y fructificación, y así se disminuye el tiempo de incubación y cosecha.

Con este proceso se produjo un doble beneficio, ya que se logró obtener cuerpo fructífero a partir de sustratos de cáscara de cacao que no son utilizados después de su descocote, además de que se consiguió una contribución con una reducción de volumen del $60 \%$ de la biomasa lignocelulósica, lo que ocasiona una menor generación de lixiviados dentro del área del cultivo. Esto comprueba la óptima capacidad del hongo de alimentarse y crecer a partir de esta biomasa vegetal y servir como agente de biorremediación para los mal llamados desechos. Por tanto, esto se constituye en un aporte a la adaptación al cambio climático, pues proporciona alternativas de uso agroindustrial y para diferentes fines.

\section{Implementación del análisis multicriterio para la evaluación de flujo de lixiviados}

En la figura 2 se muestran los resultados del flujo de lixiviados generado por la degradación orgánica en la obtención del grano de cacao después del descocote.

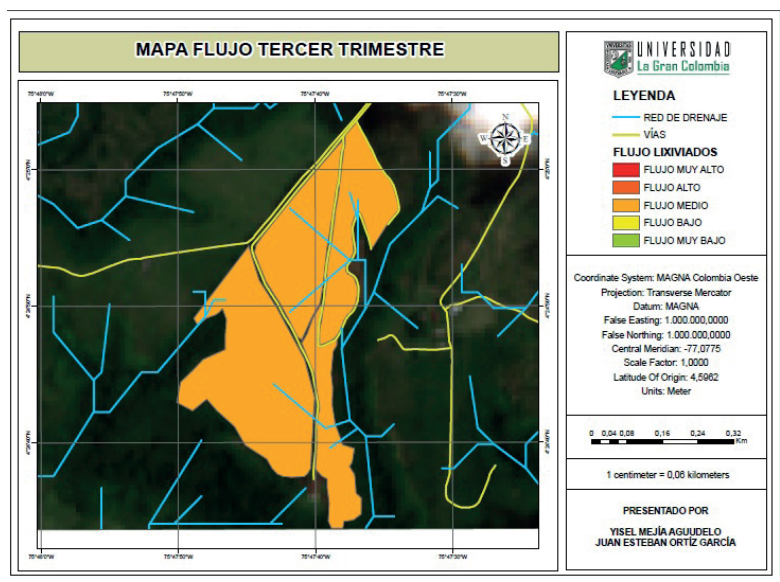

Figura 2. Flujo de lixiviados producidos en el cultivo de cacao Theobroma cacao L.

En el mapa mostrado, es posible visualizar los resultados correspondientes al flujo de lixiviados del tercer trimestre del año 2018 a partir de las tablas 1 y 2, correspondiente a la finca Paraná, donde se pueden observar las capas de los polígonos del cultivo, la red de drenaje y una imagen satelital del sector, en la que, por medio de la ponderación de relaciones espaciales, se asignó a los polígonos un valor de flujo medio de lixiviados, debido a las síntesis de la tasa de producción del cultivo junto a la precipitación. Además de esto, se puedo observar que diferentes corrientes guiadas por los canales hidrológicos se interceptan con los polígonos del cultivo; es por ello que los lixiviados se combinan con el flujo de agua, de modo que se transportan a través de él para después alcanzar el nivel freático del suelo. 
"El agua que penetra a través del terreno [..] De esta agua infiltrada, una parte es retenida por el suelo, hasta alcanzar el valor máximo que puede retener el suelo, es decir, la capacidad de campo, y una vez superada esta retención máxima, el resto del agua desciende por gravedad hasta el nivel freático, es decir hasta la zona saturada. Esta parte del agua infiltrada en el terreno que llega a las aguas subterráneas" [37].

De esta manera, aunque el cultivo esté distanciado de un cuerpo de agua, a través de este ciclo los lixiviados se verterán en las corrientes hídricas. Además de esto, cabe aclarar que el mapa 1 es una aproximación al comportamiento general del sector de estudio, debido principalmente a la falta de información de producción del cultivo Theobroma cacao L. Sin embargo, correspondió una influencia significativa de los lixiviados sobre el área del sector. Con base en la información espacial del sector agrícola dedicado al cultivo de cacao Theobroma cacao L., se desarrolló un SIG con la capacidad informativa de resolver diferentes dudas frente al cultivo, el cual permite visualizar cada polígono a partir de las variables antes mencionadas (polígonos del cultivo, la red de drenaje) y generar información con un rango espacial más definido en la síntesis de flujo de lixiviado asignado a cada polígono, correspondiente a cada atributo por analizar. A su vez, indica que cuanto más aumente la tasa de producción, mayor será la cantidad de residuos que generan lixiviados; paralelamente, cuanto mayor sea la magnitud de las precipitaciones, el arrastre de lixiviados también será más significativo. Esto muestra que la tasa de flujo de lixiviados depende de la magnitud de los valores cualitativos de la precipitación y la producción, presentes en el lugar de estudio.

El análisis multicriterio generó un mapa donde se modela el resultado de flujo medio de lixiviados en el tercer trimestre del año 2018, el cual sirve como un indicador del impacto ambiental que estos pueden generar en las zonas a las cuales se dirigen, bien sean cuerpos de agua, cultivos o poblaciones aledañas. Además, a través de este mapa, es posible tomar decisiones adecuadas sobre esta práctica, y así cumplir los requisitos establecidos por Bolaños y Rojas. [21].

Por otro lado, se consideraron tres impactos a partir de los resultados obtenidos de la investigación. En primer lugar, se identificó el impacto social, que consiste en la mitigación tanto de problematicas sanitarias en los cuerpos de agua cercanos al cultivo como de la probable aparición de enfermedades que puedan afectar el cultivo mismo y a la comunidad aledaña.

En segundo lugar, se encontró un impacto económico, dado que se presenta una reducción de gastos al evitar que se produzcan las enfermedades como la Monilia, que pueda generar daños en el cultivo. Finalmente, el productor de cacao puede cultivar el cuerpo fructífero Pleurotus ostreatus, para tener un ingreso adicional; al mismo tiempo, podría vender el residuo como sustrato a un productor dedicado al cultivo de este tipo de hongo.

\section{Conclusión}

La caracterización fisicoquímica del residuo vegetal (cáscara de cacao) permite establecer que esta biomasa cuenta con los componentes requeridos para ser empleada en alternativas de biorremediación, como se puede evidenciar en los porcentajes de lignina y celulosa, los cuales reportaron valores de $45,23 \pm 0,184$ y $30,70 \pm$ 0,753 , respectivamente. Estas variables permiten considerar este residuo como sustrato para el desarrollo de cuerpos fructíferos, pues se logró una aparición de primordios a los 16 días, con una reducción del volumen del sustrato del $60 \%$, lo cual ocasiona una menor generación de lixiviados dentro del área del cultivo. Esto se encuentra asociado al análisis multicriterio, el cual indicó el comportamiento general del flujo de lixiviados. Al respecto se representaron de manera gráfica los impactos que producen los lixiviados sobre el ambiente, lo cual proporciona posibles opciones para la toma de decisiones oportunas sobre el cultivo de cacao.

\section{Referencias bibliográficas}

[1] Gonzales Bell J. Producción de cacao evidencia un crecimiento promedio de 6,48 $\%$ en los últimos 10 años (sitio en internet). Agronegocios. Disponible en: https://www. agronegocios.co/agricultura/produccion-decacao-evidencia-un-crecimiento-promedio-de648-en-los-ultimos-10-anos-2813684. Acceso el 18 de Mayo del 2019

[2] Fedecacao. Economía nacional (sitio en internet). Fedecacao. Disponible en: https:// www.fedecacao.com.co/portal/index.php/ es/2015-02-12-17-20-59/nacionales. Acceso 
el 20 de Junio del 2019.

[3] Martínez C. 10 Principales Actividades Económicas en Colombia (sitio en internet). Lidefer. Disponible en: https://www.lifeder.com/ actividades-economicas-colombia/. Acceso el 20 de Diciembre del 2018.

[4] Jaimes Suarez Y, Aranzazu Hernandez F. Manejo de las enfermedades del cacao (Theobroma cacao L.) en Colombia, con énfasis en monilia (Moniliophthora roreri). Colombia: Corpoica; 2010.

[5] Catie. Cuánto cacao produce un árbol en toda su vida. Disponible en: www.catie.ac.cr/ nicaragua/es/76-cuanto cacao-produce-unarbol-en-toda-su-vida.htoneladas métricasl. Acceso el 21 de Junio del 2019.

[6] Najera H. Lixiviados ¿Qué son, cómo se clasifican? (Parte 1). México: UNICACH; 2003.

[7] Ortiz K, Álvarez L. Efecto del vertimiento de subproductos del beneficio de cacao (Theobroma cacao L.) sobre algunas propiedades químicas y biológicas en los suelos de una finca cacaotera, municipio de Yaguar. Colombia: Universidad de Caldas; 2015.

[8] Guillén G, Marquez F, Sánchez J. Producción de biomasa y enzimas ligninolíticas por Pleurotus ostreatus en cultivo sumergido. México: Departamento de Acuicultura; 1998.

[9] Gómez M, Montes M. Manejo de Nematodos Endoparásitos: Proyecciones Futura. Colombia: Food and Agriculture Organization of the United Nations; 2016.

[10] Rodríguez C, Jaramillo N. Cultivo de hongos comestibles del género Pleurotus sobre residuos agrícolas de la zona cafetera. Colombia: Centro Nacional de Investigación de Café-Cenicafé; 2005.

[11] Prieto J, Martínez J. Uso De La Simulación En Cartografía: Conceptos Básicos Y Aplicaciones, Mapping Interactivo. Universidad de Jaén-España. Disponible en: http://www. mappinginteractivo.com/plantilla-ante.asp?id_ articulo=69. Acceso el 23 de febrero del 2019.

[12]Norma NMX. Protección al ambientecontaminación del suelo-residuos sólidosdeterminación del ph-método potenciométrico: UNINET; 1984.

[13]Norma AOAC. Official methods of analysis. Humedad en plantas. 20.013.USA: AOAC International; 1980.

[14] Norma TAPPI. T 211 om-02 Ash in wood, pulp, paper and paperboard:combustion at $525^{\circ} \mathrm{C}$.
USA: TAPPI; 2006.

[15] Norma AOAC. Método Oficial 962.09. Fiber (Crude) in Animal Feed and Pet Food. USA: AOAC A Internacional; 2002.

[16] Norma NTC. Determinación de contenido de grasa. Colombia: ICONTEC; 1972.

[17] TAPPI. TAPPI t222 Acid-insoluble lignin in wood and pulp. USA: TAPPI; 2002.

[18] Norma NTC. Pulpas para papel. Método para determinar las celulosas alfa, beta y gama. Colombia: ICONTEC; 1973.

[19] Toledo, M. Residuos de maíz y quinua como potenciales sustratos para el cultivo de hongos comestibles Pleurotus ostreatus (Trabajo de grado). Chimborazo, Ecuador: Escuela superior politécnica de Chimborazo; 2008.

[20] García L, Duque A, Padilla L, González L. Residuos orgánicos domésticos como sustrato para la producción de hongos Pleurotus ostreatus. Rev. Fac. Nal. Agr. 2014;67(2):11941196.

[21]Bolaños D, Rojas A. Riesgos, amenazas y vulnerabilidades de los sistemas de información geográfica. (Trabajo de grado). Bogotá, Colombia: Universidad Católica de Colombia; 2013.

[22]ULPGC. Descomposición de la materia orgánica. España: ULPGC;SF.

[23] Torres Y. Caracterización de biomasa lignocelulósica (Theobroma cacao L.) para su uso en la obtención de etanol por vía fermentativa (Trabajo de grado). Bucaramanga, Colombia: Universidad Santo Tomás; 2016.

[24] Sangronis Elba, Soto M, Valero Y, Buscema Ignacio. Cascarilla de cacao venezolano como materia prima de infusiones. Caracas: Universidad Simón Bolívar; 2014.

[25]Villamizar Jaimes AR, López Giraldo LJ. Cáscara de cacao fuente de polifenoles y fibra: simulación de una planta piloto para su extracción. Respuestas. 2017;(22):75-83.

[26] Grossi GV, Ohaco Domínguez EH, Michelis A. Determinación de fibra dietética total, soluble e insoluble en hongos comestibles de cultivo Pleurotus ostreatus. Argentina: Universidad nacional del Comahue; sf.

[27]Gómez E, Ríos L, Peña J. Efecto del Pretratamiento de Biomasa Maderera en el Rendimiento a Etanol. Inf. Tecnol. 2013;24(5):113-122.

[28] Song B. Manual del cultivador de Hongos 1, cultivo del hongo ostra. Corea del Sur: MushWorld; 2005. 
[29]Chávez Sifontes M, Domine ME. Lignina, estructura y aplicaciones: métodos de despolimerización para la obtención de derivados aromáticos de interés industrial. Av. Cienc. Ing. 2013;4(4):15-46.

[30]Baena LM, García Cardona NA. Obtención y caracterización de fibra dietaria a partir de cascarilla de las semillas tostadas de Theobroma cacao L. de una industria chocolatera colombiana. Pereira, Colombia: Universidad Tecnológica de Pereira; 2012.

[31] Guzmán G, Mata G, Soto Velazco C. El cultivo de hongos comestibles, con especial atención a especies tropicales y subtropicales en esquilmos y residuos agroindustriales. 1 ed. México: Instituto Politécnico Nacional; 1993.

[32] Benavides Calvache, OL. Aprovechamientos de residuos lignocelulósicos. Juan de Pasto, Colombia: Universidad de Nariño; 2013.

[33] Cândida Rabelo S. Avaliação de desempenho do pré-tratamento com peróxido de hidrogênio alcalino para a hidrólise enzimática de bagaço de cana-de-açúcar. Campinas, Brasil: Universidade Estadual de Campinas; 2007.

[34]Vargas PS, Hoyos JL, Mosquera SA. Uso de hojarasca de roble y bagazo de caña en la producción de Pleurotus ostreatus. BSAA. 2012;(10): 136-145.

[35]Rios MP, Hoyos JL, Mosquera SA. Evaluación de los parámetros productivos de la semilla de Pleurotus ostreatus propagada en diferentes medios de cultivo. BSAA. 2010;(8):86-94.

[36] Sánchez Vázquez JE, Royse DJ. La biología y el cultivo de Pleurotus spp. 1 ed. México: Noriega Editores; 2001.

[37] Fundación Centro Internacional de Hidrología Subterránea. Infiltración Y Escorrentía. España: Fundación Centro Internacional de Hidrología Subterránea: España; sf. 